\title{
Impact of Long-Lasting Insecticidal Nets and Indoor Residual Sprayings on Susceptibility of Anopheles gambiae (Diptera: Culicidae) in Western Côte d'Ivoire
}

\author{
Mahama Touré, $^{1,2}$ Pierre Carnevale, ${ }^{3}$ and Fabrice Chandre ${ }^{3}$ \\ ${ }^{1}$ Laboratoire de Génétique, Université Lorougnon Guédé de Daloa, Daloa BP 150, Cote d'Ivoire \\ ${ }^{2}$ Institut de Recherche pour le Développement (IRD), Abidjan 15 BP 917, Cote d'Ivoire \\ ${ }^{3}$ UMR 224 (MIVEGEC), Institut de Recherche pour le Développement (IRD), 911 Avenue Agropolis, BP 64501, \\ 34394 Montpellier Cedex 5, France
}

Correspondence should be addressed to Mahama Touré; mahamatoure@yahoo.fr

Received 13 October 2012; Accepted 15 November 2012

Academic Editors: V. Konjufca, Y. Lai, M. Ramharter, and S. Yoshida

Copyright (C) 2013 Mahama Touré et al. This is an open access article distributed under the Creative Commons Attribution License, which permits unrestricted use, distribution, and reproduction in any medium, provided the original work is properly cited.

Pyrethroid insecticides resistance in several Anopheles species represents a threat for the success of malaria vector control based on using treated nets and indoor sprayings. Impact of the long-lasting insecticidal net (LLIN) of Permanet type and indoor residual spraying (IRS) with deltamethrin on the levels of susceptibility to pyrethroids and DDT were investigated in field populations of Anopheles gambiae in 12 villages located in the Danané area in Western Côte d'Ivoire. From May 2001 to July 2002, adult An. gambiae reared from larvae collected from these villages were tested with $0.05 \%$ deltamethrin, $1 \%$ permethrin, and $4 \%$ DDT impregnated papers using WHO standard test kits. Knockdown times for $50 \%$ of adult mosquitoes $\left(\mathrm{kdT}_{50}\right)$ of field specimens from LLINs, untreated nets, IRS, and control villages significantly differed from those of the Kisumu susceptible strain. However, the resistance ratios of the $\mathrm{kdT}_{50}$ were very low. $\mathrm{KdT}_{50}$ did not significantly differ with all treatments, chemicals (LLINs and IRS), physical (untreated nets), and control. The mortalities were above $80 \%$ indicating susceptible populations of An. gambiae in the Danané area. There was no significant difference between mortality rates from the different treatments.

\section{Introduction}

Malaria vector control aims to prevent the transmission of Plasmodium parasites by using essentially treated nets and/or indoor residual sprayings with insecticides (IRS). Many malaria vector control projects based upon sprayings were stopped because resistance to insecticides occurred in malaria mosquito populations [1] such as the Anopheles gambiae complex Giles 1902 (Diptera: Culicidae), a major malaria vector in Africa. Meanwhile, insecticide treated nets (ITNs) have proved their efficacy in reducing malaria morbidity and mortality $[2,3]$, but low rates of insecticide retreatment of bed nets were a critical issue for the success of campaigns largely based on ITNs [4]. Thus, long-lasting insecticidal nets (LLINs) have been proposed and reported to protect against mosquitoes for several years [5].
To date, five (5) pyrethroids ( $\alpha$-cypermethrin, cyfluthrin, deltamethrin, $\lambda$-cyhalothrin, and permethrin) and one pseudopyrethroid (etofenprox) insecticides are recommended for net impregnation [6]. These insecticides are advocated for vector control particularly in public health because of their quick knockdown and lethal effects on mosquitoes at low concentrations [7]. They also produce excito-repellent effects on mosquitoes. Insecticide-treated nets significantly reduce indoor resting densities of malaria vectors and sporozoites rates [8]. However, the recent evolution of pyrethroid resistance in several Anopheles species represents a threat for the success of malaria vector control. A lot of researches have reported this type of resistance in several South Sahara African populations of An. gambiae sensu stricto [9, 10]. The occurrence of this resistance induces a significant reduction in mosquito mortality after exposure to permethrin and lower 
knockdown effects of deltamethrin and $\lambda$-cyhalothrin [11, 12]. Investigations on the target site of action for pyrethroids show that the knockdown resistance $(\mathrm{kdr})$ is mainly due to a single-point mutation in the gene coding for the structure of the voltage-gated sodium channel, resulting in a change in amino acid from leucine to phenylalanine (L1014F) [13] or from leucine to serine (L1014S) [14]. This mutation confers cross-resistance to a wide range of pyrethroids and DDT [11]. It was initially detected in the $S$ molecular form of $A n$. gambiae s.s. from tropical Savanna areas $[1,12,15]$. In Benin and Ghana, this mutation has also been reported in the $\mathrm{M}$ molecular form $[16,17]$ and is now spreading in $M$ form populations from dry Savanna areas [18].

The cytochrome $\mathrm{P}_{450}$ monooxygenase-enzymes have also been reported to induce resistance to pyrethroids [19]. Indeed, over production of these enzymes has been associated with pyrethroid resistance in both An. albimanus and An. gambiae species [20]. Other results have suggested that high levels of oxidase enzymes were responsible for detoxification of pyrethroids in An. funestus in Northern Natal Kwazulu in South Africa [21]. More recent studies have reported six genes whose expression levels are associated with pyrethroid metabolic resistance of A. gambiae from West Africa [22]. Three of these six genes were overexpressed in both sexes of the resistant strain, two cytochrome $\mathrm{P}_{450}$ (CYP6Z2 and CYP6M2) and a superoxide dismutase (SOD3B). In females of the resistant strain, a carboxylesterase (COEJHE5E) and a GST (GSTS1-2) were also found to be overexpressed while another $\mathrm{P}_{450}$ (CYP6Z3) was overexpressed in the resistant males only.

The current survey was carried out from May 2001 to July 2002 in villages located in Western Côte d'Ivoire to investigate the impact of both LLINs of Permanet type and IRS with deltamethrin on the levels of susceptibility to pyrethroids and DDT of field An. gambiae populations.

\section{Material and Methods}

2.1. Treatments in Study Sites. The study involved 12 villages in the area of Danané city $\left(7^{\circ} 15^{\prime} \mathrm{N}, 8^{\circ} 9^{\prime} \mathrm{W}, 365 \mathrm{~m}\right.$ altitude) in western Côte d'Ivoire. Daily average temperature during the trial period was around $25^{\circ} \mathrm{C}$ with the minimum and maximum being $20^{\circ} \mathrm{C}$ and $34^{\circ} \mathrm{C}$, respectively. Nets were rarely used in this area before the trial. Villages were grouped in three for each treatment.

Permanet is a LLIN made of polyester net and treated at the factory level with deltamethrin at a concentration of 55 a.i. $\mathrm{mg} / \mathrm{m}^{2}$. The dosage is two times higher than that of hand-treated nets $\left(25\right.$ a.i. $\left.\mathrm{mg} / \mathrm{m}^{2}\right)$. Permanet is reported to be active for up to 20 standard washes, and its biological activity lasts as long as the net itself, 3 to 5 years. In this study, three villages, Finneu, Bouenneu, and Danta, were provided with LLINs, with each sleeping unit receiving a bed net. Thus, one thousand eight hundred and five $(1,805)$ LLINs were distributed in May 2001. These nets represented a total amount of $1,290.575$ a.i.g $\left(55\right.$ a.i. $\left.\mathrm{mg} / \mathrm{m}^{2} \times 13 \mathrm{~m}^{2} \times 1,805\right)$ of the active ingredient used to cover the three villages. One thousand six hundred and ninety-five $(1,695)$ nets of the same material without insecticide were also given to sleeping units in three other villages, Seileu, Peuple, and Zoleu, which served as controls to the villages provided with LLINs.

IRS with deltamethrin WG 20 a.i. $\mathrm{mg} / \mathrm{m}^{2}$ on walls of every house was done in three other villages: Yotta, Gbontégleu, and Vétouo. The spraying operation was repeated six months later in November with the same insecticide formulation at the same concentration, in these same villages. The amounts of deltamethrin used for IRS in May and in November 2001 were 755 and 737 a.i.g, respectively. The first IRS covered $37,510 \mathrm{~m}^{2}$, while the second one covered $32,010 \mathrm{~m}^{2}$. Three other villages, Bepleu, Biétouo, and Méantouo, where no intervention was carried out, served as controls.

2.2. Nets and Insecticides Provision. The nets were made of white multifilament polyester fabric ( 75 deniers, 156 meshes, $12 \times 13$ holes/in ${ }^{2}$, Vestergaard Frandsen A/S, Copenhagen, Denmark). IRS was done using deltamethrin (K-Othrine) WG 250 (25\% ww, Sanofi-Aventis formerly Aventis, Paris, France).

2.3. An. gambiae Collections. Larvae of An. gambiae were collected in paddy fields and puddles from May 2001 to July 2002 at three-month intervals in all the 12 villages. Larvae were reared locally until adult stages. Bioassays were carried out using three-to-five-day old nonblood fed females. Test mosquitoes were identified morphologically, and only females of An. gambiae were selected for bioassays. The "Kisumu" strain of An. gambiae reared in our insectary was used as susceptible reference.

2.4. Bioassays. Bioassays were carried out with $\mathrm{WHO}$ insecticide susceptibility test kits for adult mosquitoes using $0.05 \%$ deltamethrin, $1 \%$ permethrin, and $4 \%$ DDT impregnated papers. These are recommended dosages for diagnostic tests of susceptibility to insecticides [23] after a one hour holding period.

Papers were impregnated in our laboratory according to $\mathrm{WHO}$ protocol using Dow Corning 556 silicone oil as a carrier [23]. Insecticides with the recommended active ingredients were provided by Agrevo, Berkhamsted, U.K.

The numbers of mosquitoes knocked down were recorded at 10-min intervals during a 60-min exposure. Mortality rates were determined after 24 hours holding period with a supply of $10 \%$ honey solution. All experiments were carried out at a temperature and humidity of $25^{\circ} \mathrm{C}$ and $70-80 \% \mathrm{RH}$, respectively.

2.5. Data Analysis. Data were analysed using log-probit software based on the Finney model [24]. Knockdown times for $50 \%$ individuals $\left(\mathrm{kdT}_{50}\right)$ were determined per bioassay. Ratios of the $\mathrm{kdT}_{50}$ of field An. gambiae over those of the Kisumu susceptible strain $\left(\mathrm{RR}_{50}\right)$ were determined.

Mortality rates were calculated and analysed according to the WHO criteria in order to determine whether samples were susceptible or resistant [23]. 
TABLE 1: Variation of the knockdown times for 50\% individuals of field An. gambiae from long-lasting insecticidal nets (LLINs) of Permanet type, indoor residual sprayings (IRS), untreated nets and controls villages within 24 hours following 1 hour exposure to $0.05 \%$ deltamethrin, $1 \%$ permethrin and $4 \%$ DDT impregnated papers, $95 \%$ confidence intervals in brackets.

\begin{tabular}{|c|c|c|c|c|}
\hline Months & Status & $0.05 \%$ deltamethrin & $1 \%$ permethrin & $4 \%$ DDT \\
\hline & Kisumu & $9.4(8.5-10.2)$ & $6.3(5.7-6.9)$ & $15.4(12.8-18.5)$ \\
\hline \multirow{4}{*}{0} & LLINs & $23.7(22.7-24.8)$ & $12.0(9.3-15.4)$ & $24.6(23.5-25.8)$ \\
\hline & Untreated nets & $15.1(13.1-17.3)$ & $12.3(11.7-13.0)$ & $30.6(25.0-37.3)$ \\
\hline & IRS & $23.0(21.8-24.1)$ & $14.3(12.9-15.7)$ & $35.9(29.2-44.1)$ \\
\hline & Controls & $16.2(14.2-17.5)$ & $8.3(7.4-9.2)$ & $17.0(12.9-22.5)$ \\
\hline \multirow{4}{*}{3} & LLINs & $17.9(16.5-19.4)$ & $15.1(13.1-17.6)$ & - \\
\hline & Untreated nets & $16.4(15.2-17.7)$ & - & - \\
\hline & IRS & $15.7(12.0-20.5)$ & - & - \\
\hline & Controls & $13.1(11.9-14.3)$ & $13.4(10.7-15.7)$ & - \\
\hline \multirow{4}{*}{6} & LLINs & $21.9(20.5-23.2)$ & $15.3(13.7-16.9)$ & $41.5(39.3-43.8)$ \\
\hline & Untreated nets & $19.6(18.6-20.7)$ & $13.4(12.1-14.7)$ & $27.1(25.2-29.1)$ \\
\hline & IRS & $20.5(19.4-21.6)$ & $12.3(9.0-16.7)$ & $39.7(36.3-43.3)$ \\
\hline & Controls & $20.8(19.2-22.5)$ & $12.9(11.7-14.3)$ & - \\
\hline \multirow{4}{*}{12} & LLINs & $15.3(14.6-16.1)$ & $12.9(11.6-14.4)$ & $24.0(21.9-26.1)$ \\
\hline & Untreated nets & $15.6(13.7-17.6)$ & $9.5(8.5-10.5)$ & $33.3(28.8-38.5)$ \\
\hline & IRS & $14.3(13.4-15.2)$ & $11.2(9.6-13.0)$ & $27.0(24.9-29.3)$ \\
\hline & Controls & $13.5(12.6-14.4)$ & $11.9(10.1-13.9)$ & $9.0(5.9-11.7)$ \\
\hline \multirow{4}{*}{15} & LLINs & $10.5(9.4-11.4)$ & $9.0(7.2-11.2)$ & $25.2(22.1-28.7)$ \\
\hline & Untreated nets & $14.6(13.7-15.4)$ & $7.8(7.2-8.3)$ & $19.8(16.8-23.3)$ \\
\hline & IRS & $13.0(12.2-13.8)$ & - & - \\
\hline & Controls & $12.5(11.8-13.1)$ & $7.9(7.3-8.5)$ & $23.1(20.9-25.6)$ \\
\hline
\end{tabular}

The Jonckheere-Terpstra nonparametric test was used to compare variation in the $\mathrm{kdT}_{50}$ of samples. A Cox proportional hazard model was then used to compare the levels of susceptibility of field An. gambiae based on mortality rates. Both the Jonckheere-Terpstra test and the Cox model were calculated using SPSS software version 11.5.

\section{Results}

3.1. $K d T_{50}$ and Mortality Rates of the Kisumu Strain. $\mathrm{KdT}_{50}$ of the Kisumu susceptible strain were $9.4 \mathrm{~min}, 6.3 \mathrm{~min}$, and 15.4 min with $0.05 \%$ deltamethrin, $1 \%$ permethrin, and $4 \%$ DDT, respectively, while mortality rates after the 24-hour holding period were $100 \%$ (Tables 1 and 2).

3.2. $\mathrm{KdT}_{50}$ and Mortality Rates of Field An. gambiae in May 2001. The $\mathrm{kdT}_{50}$ of field collected An. gambiae from LLINs, untreated nets, IRS and control villages were 23.7, $15.1,23$ and $16.2 \mathrm{~min}$, respectively with deltamethrin, 12, $12.3,14.3$ and $8.3 \mathrm{~min}$, respectively with permethrin and 24.6, 30.6, 35.9 and $17 \mathrm{~min}$, respectively with DDT (Tables 1 and 2). The $\mathrm{kdT}_{50}$ values were different before treatments (LLINs, untreated nets, IRS, and control) on month 0 with all insecticides.

Mortality rates of field An. gambiae were $100 \%$ in LLINs, untreated nets, and control villages and 95\% in IRS villages with deltamethrin. Mortality rates were $100 \%$ in villages given untreated nets and control villages, 99\% in LLINs villages, and $91 \%$ in IRS-treated villages with permethrin, while the rates with DDT were $100 \%$ in villages given untreated bed nets and control villages, $98 \%$ in villages given LLINs, and 96\% in IRStreated villages.

3.3. Variations of $k d T_{50}$ and Mortality Rates of Field An. gambiae during the Trial. The $\mathrm{kdT}_{50}$ values with deltamethrin from all villages, with or without insecticide were around 15 min (Figure $1(\mathrm{a})$ ). Using permethrin, $\mathrm{kdT}_{50}$ values were around $10 \mathrm{~min}$ (Figure $1(\mathrm{~b})$ ) while values remained high throughout the trial with DDT (Figure $1(\mathrm{c})$ ). The $\mathrm{kdT}_{50}$ values for all three insecticides for all the different populations were significantly different from those observed with the Kisumu susceptible strain $(P<0.001)$. However, the $\mathrm{RR}_{50}$ of these $\mathrm{kdT}_{50}$ were 1.1-2.7 compared to the susceptible reference strain, indicating that differences were low (Table 3).

The $\mathrm{kdT}_{50}$ induced by both $0.05 \%$ deltamethrin and $1 \%$ permethrin slightly decreased from the $6^{\text {th }}$ month to the 15 th month without any significant difference according to the different treatments during the whole trial $(P=$ 0.130 and $P=0.174$, resp.). As with deltamethrin and permethrin, there was no significant difference between the $\mathrm{kdT}_{50}$ observed with $4 \%$ DDT $(P=0.177)$.

Mortality rates were high, $95 \%-100 \%$ with deltamethrin (Figure 2(a)), 91-100\% with permethrin (Figure 2(b)), and $83-100 \%$ with DDT (Figure 2(c)). Even though overall mortality rates were $>80 \%$, a few samples showed mortalities $<98 \%$. There was no significant difference between the mortality rates of samples tested with deltamethrin and 
TABLE 2: Variation of the mortality rates of field An. gambiae from long-lasting insecticidal nets (LLINs) of Permanet type, indoor residual sprayings (IRS), untreated nets, and controls villages within 24 hours following 1 -hour exposure to $0.05 \%$ deltamethrin, $1 \%$ permethrin, and $4 \%$ DDT impregnated papers.

\begin{tabular}{|c|c|c|c|c|}
\hline Months & Status & $\begin{array}{c}0.05 \% \\
\text { deltamethrin }\end{array}$ & $\begin{array}{c}1 \% \\
\text { permethrin }\end{array}$ & $4 \% \mathrm{DDT}$ \\
\hline & Kisumu & 100 & 100 & 100 \\
\hline \multirow{4}{*}{0} & LLINs & 100 & 99 & 98 \\
\hline & Untreated nets & 100 & 100 & 100 \\
\hline & IRS & 95 & 91 & 96 \\
\hline & Controls & 100 & 100 & 100 \\
\hline \multirow{4}{*}{3} & LLINs & 100 & 100 & - \\
\hline & Untreated nets & 100 & - & - \\
\hline & IRS & 100 & - & - \\
\hline & Controls & 100 & 100 & - \\
\hline \multirow{4}{*}{6} & LLINs & 100 & 99 & 98 \\
\hline & Untreated nets & 98 & 100 & 99 \\
\hline & IRS & 99 & 98 & 99 \\
\hline & Controls & 99 & 100 & - \\
\hline \multirow{4}{*}{12} & LLINs & 99 & 100 & 89 \\
\hline & Untreated nets & 97 & 99 & 95 \\
\hline & IRS & 99 & 96 & 83 \\
\hline & Controls & 99 & 96 & 83 \\
\hline \multirow{4}{*}{15} & LLINs & 98 & 100 & 94 \\
\hline & Untreated nets & 100 & 100 & 99 \\
\hline & IRS & 100 & - & - \\
\hline & Controls & 100 & 100 & 98 \\
\hline
\end{tabular}

permethrin $(P=0.129$ and $P=0.344$, resp. $)$ and with DDT $(P>0.05)$.

3.4. $K d T_{50}$ and Mortality Rates of Field An. gambiae at the End of the Trial. With deltamethrin, the $\mathrm{kdT}_{50}$ were 10.5, 14.6, 13, and $12.5 \mathrm{~min}$ from LLINs, untreated nets, IRS, and control villages, respectively. The $\mathrm{kdT}_{50}$ from LLINs, untreated nets, and control villages were 9.0, 7.8 and $7.9 \mathrm{~min}$, respectively, with permethrin and $25.2,19.8$, and $23.1 \mathrm{~min}$, respectively, with DDT.

Mortality rates were $100 \%$ from all villages with permethrin and from untreated nets, IRS, and control villages with deltamethrin. Mortality rates were $98 \%$ in LLINs villages with deltamethrin. With DDT, mortality rates were $94 \%$, 99\%, and 98\% in LLINs, untreated nets, and control villages, respectively.

\section{Discussion}

The $\mathrm{kdT}_{50}$ of field An. gambiae exposed to $0.05 \%$ deltamethrin, $1 \%$ permethrin, and 4\% DDT impregnated papers that were almost two times higher than those of the Kisumu susceptible strain. This result indicates susceptibility to insecticides of these populations which could be due to a
TABLE 3: Variation of ratios of the knockdown times for $50 \%$ individuals $\left(\mathrm{RR}_{50}\right.$ with $\left.95 \% \mathrm{CI}\right)$ of field An. gambiae from longlasting insecticidal nets (LLINs) of Permanet type, indoor residual sprayings (IRS), untreated nets, and controls villages over the knockdown times for $50 \%$ individuals of the Kisumu susceptible strain exposed for 1 hour to $0.05 \%$ deltamethrin, $1 \%$ permethrin, and $4 \%$ DDT impregnated papers.

\begin{tabular}{|c|c|c|c|c|}
\hline Months & Status & $\begin{array}{c}0.05 \% \\
\text { deltamethrin } \\
\end{array}$ & $\begin{array}{c}1 \% \\
\text { permethrin }\end{array}$ & $4 \% \mathrm{DDT}$ \\
\hline \multirow{4}{*}{0} & LLINs & 2.5 & 1.9 & 1.6 \\
\hline & Untreated nets & 1.6 & 2.0 & 2.0 \\
\hline & IRS & 2.4 & 2.3 & 2.3 \\
\hline & Controls & 1.7 & 1.3 & 1.1 \\
\hline \multirow{4}{*}{3} & LLINs & 1.9 & 2.4 & - \\
\hline & Untreated nets & 1.8 & - & - \\
\hline & IRS & 1.7 & - & - \\
\hline & Controls & 1.4 & 2.1 & - \\
\hline \multirow{4}{*}{6} & LLINs & 2.3 & 2.4 & 2.7 \\
\hline & Untreated nets & 2.1 & 2.1 & 1.8 \\
\hline & IRS & 2.2 & 2.0 & 2.6 \\
\hline & Controls & 2.2 & 2.0 & - \\
\hline \multirow{4}{*}{12} & LLINs & 1.6 & 2.0 & 1.6 \\
\hline & Untreated nets & 1.7 & 1.5 & 2.2 \\
\hline & IRS & 1.5 & 1.8 & 1.8 \\
\hline & Controls & 1.4 & 1.8 & 0.6 \\
\hline \multirow{4}{*}{15} & LLINs & 1.1 & 1.4 & 1.6 \\
\hline & Untreated nets & 1.6 & 1.2 & 1.3 \\
\hline & IRS & 1.4 & - & - \\
\hline & Controls & 1.3 & 1.3 & 1.5 \\
\hline
\end{tabular}

lower frequency of resistant individuals since the knockdown time has been reported to be an indicator for early detection of knockdown resistance [11]. No significant differences were observed between the $\mathrm{kdT}_{50}$ of field An. gambiae from the different treatments and control areas suggesting that neither LLINs nor IRS with deltamethrin induced any change of the susceptibility of populations during the whole trial period. These results are consistent with studies performed in Western Kenya, where resistance levels of field mosquitoes were reported to remain low two years after implementation of permethrin pretreated nets [8].

The mortality rates revealed a susceptibility of An. gambiae to $0.05 \%$ deltamethrin, $1 \%$ permethrin, and $4 \%$ DDT in this area of Côte d'Ivoire, and this did not change during the 15 months study period of insecticide use $(P>0.05)$. Indeed, unpublished data of research performed in these study sites showed that mosquitoes belonged mainly to the $\mathrm{M}$ molecular form of An. gambiae s.s. that did not carry the kdr mutation conferring cross-resistance to pyrethroids and DDT. Thus, even though we did not carry out molecular genotyping of the specimens collected from this area, our expectation is that they will belong to this molecular form of An. gambiae s.s. Subsequently, the high mortality rates observed may be 


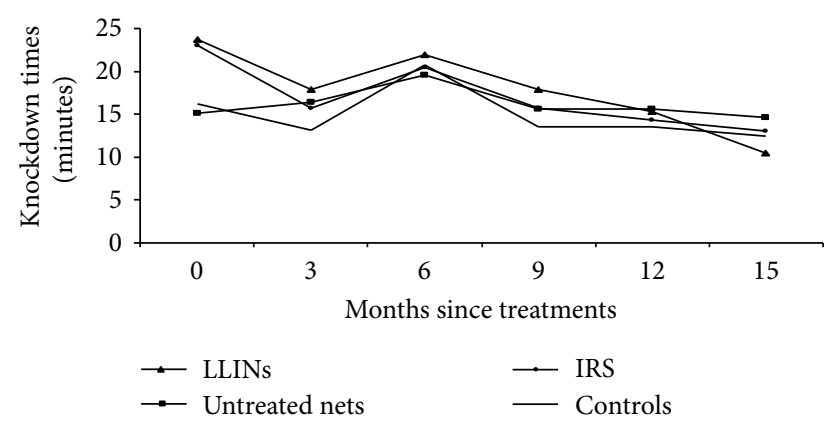

(a)

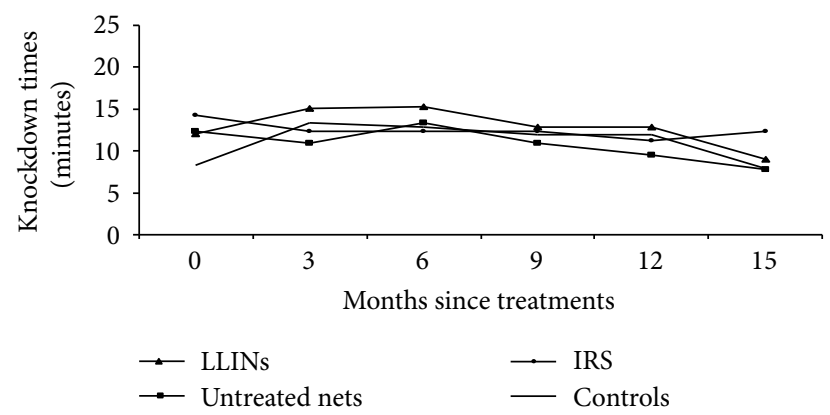

(b)

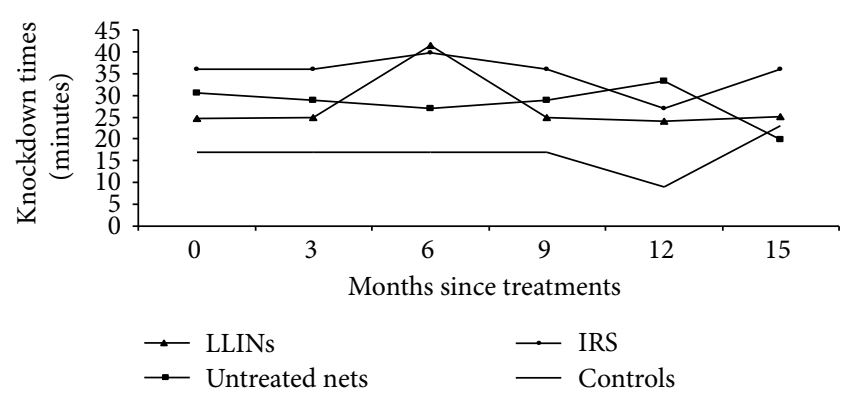

(c)

FIgURE 1: Variation of the knockdown times for 50\% individuals $\left(\mathrm{kdT}_{50}\right)$ of field An. gambiae from long-lasting insecticidal nets (LLINs) of Permanet type, indoor residual sprayings (IRS), untreated nets, and controls villages exposed for 1 hour to (a) $0.05 \%$ deltamethrin, (b) $1 \%$ permethrin, and (c) $4 \%$ DDT impregnated papers.

likely due to this high proportion of susceptible $M$ form mosquitoes.

However, the observation that mortalities to all three insecticides slightly decreased at the beginning of the rainy season on the 12th month of the study period may indicate an increase in the frequency of the S molecular form of $A n$. gambiae s.s. The S molecular form of An. gambiae s.s. is generally associated with temporary breeding sites (puddles, wheel prints, etc.) and is known to increase in frequency in the rainy season, while the $\mathrm{M}$ molecular form is associated with permanent breeding sites such as paddy fields and irrigated rice fields [12]. The decrease in mortality, though weak during our survey period, might be likely due to individuals of the $\mathrm{S}$ molecular form that carried knockdown mutation and may be expected to increase as the rainy

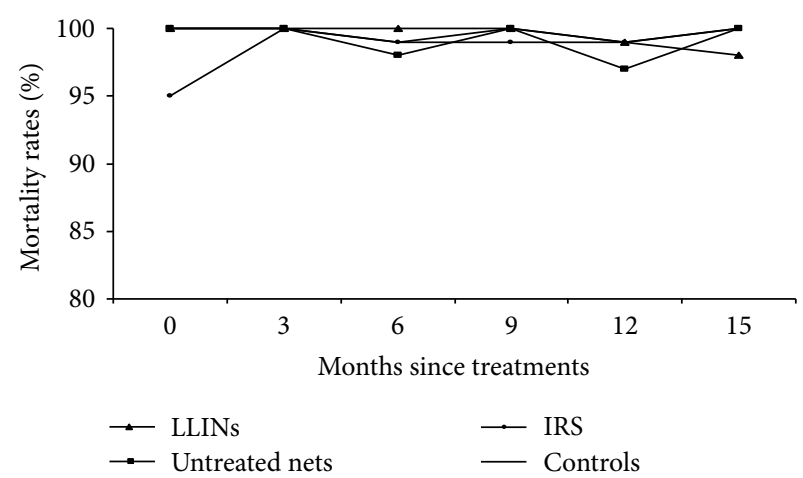

(a)

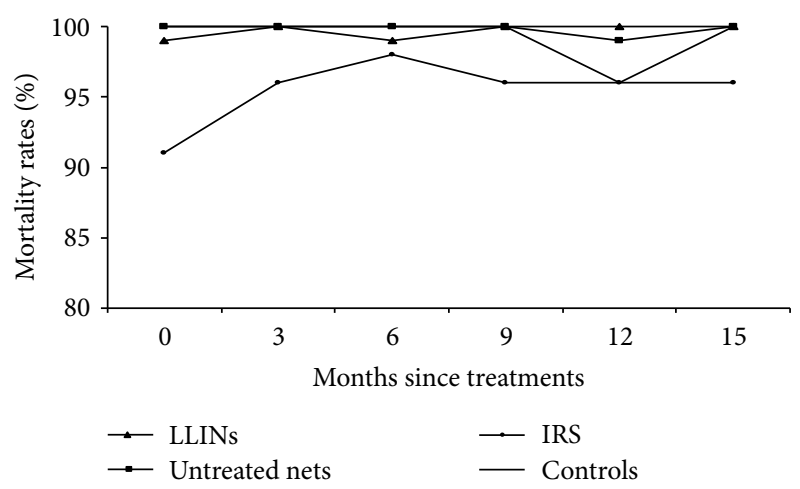

(b)

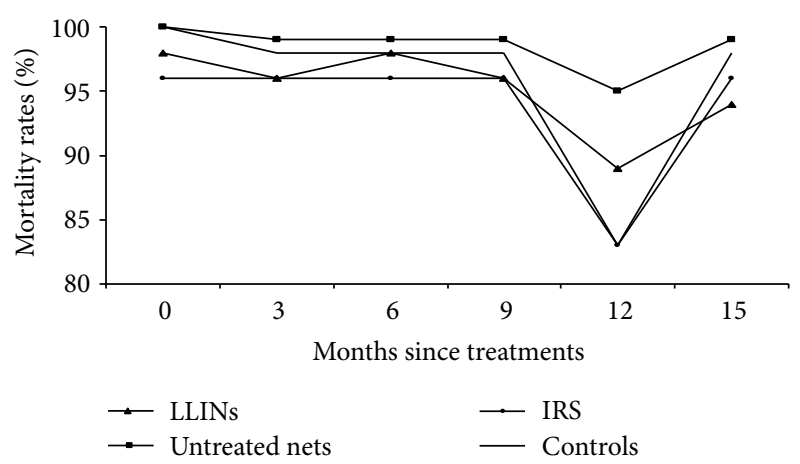

(c)

FIGURE 2: Variation of the mortality rates of field An. gambiae from long-lasting insecticidal nets (LLINs) of Permanet type, indoor residual sprayings (IRS), untreated nets, and controls villages within 24 hours following 1 hour exposure to (a) 0.05\% deltamethrin, (b) $1 \%$ permethrin, and (c) $4 \%$ DDT impregnated papers.

season progressed. In contrary, recent studies reported that $\mathrm{kdr}$ mutation was found with a frequency of $70 \%$ in the $\mathrm{M}$ molecular form of An. gambiae from the French military camp of Port Bouët in Southern Côte d'Ivoire [25].

Observed mortalities in this study were lower with DDT than with deltamethrin and permethrin. A similar phenomenon was observed in Burkina Faso, where local mosquitoes were susceptible to deltamethrin and permethrin, but resistant to DDT [12]. These low mortalities may be explained by metabolic based mechanism such as glutathione-S-transferases that confer resistance to DDT. 
Resistance development in the field has been reported to depend on the interaction of several factors, which can be classified into three categories: genetic, biological, and operational [26]. The level and the type of resistance can differ according to ecological sitting. In coastal areas of Benin in West Africa, the cross-resistance of An. gambiae s.l. to pyrethroids and DDT was explained by the presence of the $\mathrm{kdr}$ mutation at high frequency [27]. Whereas at Siguiri in Guinea Conakry in West Africa, populations of An. gambiae showed high levels of resistance to DDT, although the frequency of the $1014 \mathrm{~F} \mathrm{kdr}$ allele was only $24 \%$ [28].

In this study, the presence of few resistant specimens among susceptible populations could be taken as an indication that resistance would increase over time under selective insecticide pressure. Nevertheless, available data did not show any increase of resistance in field An. gambiae populations. Indeed, studies on larvae of An. stephensi selected with DDT for 40 generations were reported to develop a 126.6-fold increase in resistance to DDT but showed only 3.7-fold crossresistance to deltamethrin [29]. The absence or the very weak selection pressure exerted by the LLINs on resistance during this study may be due to the low operational dose of insecticide for bed net impregnation and/or repellent effects of LLINs and IRS with deltamethrin. Near Kisumu in Western Kenya, the frequencies of knockdown mutation increased only from $3 \%$ to $8 \%$ in villages where permethrin treated bed nets (ITNs) were used and the surrounding villages during 14 years study period, from 1987 to 2001 [30].

Indoor residual sprayings with deltamethrin were done only twice in 12 th month during the current study, leading to an absence or a very low selection pressure just as it was observed with the LLINs. Selection pressures may be higher where insecticides are widely used, such as in agriculture as opposed to pyrethroid-treated bed nets and IRS.

Notwithstanding, the current data shows that field $A n$. gambiae populations of Danané remained susceptible to deltamethrin, permethrin, and DDT over a 15-month period of active insecticide usage.

\section{Authors' Contribution}

The authors thank Pierre Carnevale for coordinating the paper and advising about the original records, Fabrice Chandre for useful advice about data collection, analysis, and commenting on the draft, and Mahama Touré for field sampling, experiments, data analysis and paper writing.

\section{Acknowledgments}

The authors are grateful to all the volunteers and local coordinators who have contributed to the realization of this study. Thanks to Doctors Yawson A. E. and Brown C. from the Noguchi Memorial Institute for Medical Resarch in Ghana for their technical assistance. This study was supported by the PAL+ programme and the French Institute of Research for Development (IRD, formerly ORSTOM). The authors thank Vestergaard Frandsen A/S and Aventis for providing nets and insecticides for house sprayings, respectively. The authors are grateful to Agrevo for providing insecticides for WHO tests.

\section{References}

[1] P. Carnevale and J. Mouchet, "La lutte antivectorielle au Cameroun. Passé-présent-avenir. Réflexions," Bulletin de la Société de Pathologie Exotique, vol. 94, no. 2, pp. 202-209, 2001.

[2] U. D’Alessandro, B. Olaleye, W. McGuire et al., "Mortality and morbidity from malaria in Gambian children after introduction of an impregnated bednet programme," The Lancet, vol. 345, no. 8948, pp. 479-483, 1995.

[3] B. L. Nahlen, J. P. Clark, and D. Alnwick, "Insecticide-treated bed nets," American Journal of Tropical Medicine and Hygiene, vol. 68, no. 4, pp. 1-2, 2003.

[4] J. E. Miller, S. W. Lindsay, and J. R. M. Armstrong, "Experimental hut trials of bednets impregnated with synthetic pyrethroid or organophosphate insecticide for mosquito control in the Gambia," Medical and Veterinary Entomology, vol. 5, no. 4, pp. 465-476, 1991.

[5] T. Mahama, E. J. Desiree, C. Pierre, and C. Fabrice, "Effectiveness of permanet in Côte d'Ivoire rural areas and residual activity on a knockdown-resistant strain of Anopheles gambiae," Journal of Medical Entomology, vol. 44, no. 3, pp. 498-502, 2007.

[6] World Health Organization, "Review of: IR3535; KBR3023; (RS)-methoprene $20 \%$ EC, pyriproxyfen $0.5 \%$ GR; and lambdacyhalothrin 2.5\% CS," Report of WHOPES Working Group Meeting, Document WHO/CDS/WHOPES/2001.2, Geneva, Switzerland, December 2000.

[7] D. C. Chavasse and H. H. Yap, "Chemical Methods for the Control of Vectors and Pests of Public Health Importance," WHO/CTD/WHOPES/97.2, World Health Organization, Geneva, Switzerland, 1997.

[8] J. E. Gimnig, J. M. Vulule, T. Q. Lo et al., "Impact of permethrintreated bed nets on entomologic indices in an area of intense year-round malaria transmission," American Journal of Tropical Medicine and Hygiene, vol. 68, no. 4, pp. 16-22, 2003.

[9] F. Chandre, F. Darriet, S. Manguin, C. Brengues, P. Carnevale, and P. Guillet, "Pyrethroid cross resistance spectrum among populations of Anopheles gambiae s.s. from Côte d'Ivoire," Journal of the American Mosquito Control Association, vol. 15, no. 1, pp. 53-59, 1999.

[10] T. S. Awolola, I. O. Oyewole, C. N. Amajoh et al., "Distribution of the molecular forms of Anopheles gambiae and pyrethroid knock down resistance gene in Nigeria," Acta Tropica, vol. 95, no. 3, pp. 204-209, 2005.

[11] F. Chandre, F. Darrier, L. Manga et al., "Status of pyrethroid resistance in Anopheles gambiae sensu lato," Bulletin of the World Health Organization, vol. 77, no. 3, pp. 230-234, 1999.

[12] A. Diabate, T. Baldet, F. Chandre et al., "The role of agricultural use of insecticides in resistance to pyrethroids in Anopheles gambiae s.l. in Burkina Faso," American Journal of Tropical Medicine and Hygiene, vol. 67, no. 6, pp. 617-622, 2002.

[13] D. Martinez-Torres, F. Chandre, M. S. Williamson et al., "Molecular characterization of pyrethroid knockdown resistance (kdr) in the major malaria vector Anopheles gambiae s.s.", Insect Molecular Biology, vol. 7, no. 2, pp. 179-184, 1998.

[14] H. Ranson, B. Jensen, J. M. Vulule, X. Wang, J. Hemingway, and F. H. Collins, "Identification of a point mutation in the voltage-gated sodium channel gene of Kenyan Anopheles gambiae associated with resistance to DDT and pyrethroids," Insect Molecular Biology, vol. 9, no. 5, pp. 491-497, 2000. 
[15] F. Chandre, C. Brengues, J. Dossou Yovo et al., "Current distribution of a pyrethroid resistance gene (kdr) in Anopheles gambiae complex from West Africa and further evidence for reproductive isolation of the Mopti form," Parassitologia, vol. 41, no. 1-3, pp. 319-322, 1999.

[16] M. Weill, F. Chandre, C. Brengues et al., "The kdr mutation occurs in the Mopti form of Anopheles gambiae s.s. through introgression," Insect Molecular Biology, vol. 9, no. 5, pp. 451-455, 2000.

[17] A. E. Yawson, P. J. McCall, M. D. Wilson, and M. J. Donnelly, "Species abundance and insecticide resistance of Anopheles gambiae in selected areas of Ghana and Burkina Faso," Medical and Veterinary Entomology, vol. 18, no. 4, pp. 372-377, 2004.

[18] K. R. Dabiré, A. Diabaté, L. Djogbenou et al., "Dynamics of multiple insecticide resistance in the malaria vector Anopheles gambiae in a rice growing area in South-Western Burkina Faso," Malaria Journal, vol. 7, article 188, 2008.

[19] J. G. Scott, "Cytochrome P450 monooxygenase-mediated resistance to insecticides," Journal of Pesticide Sciences, vol. 21, no. 2, pp. 241-245, 1996.

[20] J. Hemingway and H. Ranson, "Insecticide resistance in insect vectors of human disease," Annual Review of Entomology, vol. 45, pp. 371-391, 2000.

[21] B. D. Brooke, G. Kloke, R. H. Hunt et al., "Bioassay and biochemical analyses of insecticide resistance in Southern African Anopheles funestus (Diptera: Culicidae)," Bulletin of Entomological Research, vol. 91, no. 4, pp. 265-272, 2001.

[22] P. Müller, M. J. Donnelly, and H. Ranson, "Transcription profiling of a recently colonised pyrethroid resistant Anopheles gambiae strain from Ghana," BioMed Central Genomics, vol. 8, article 36, 2007.

[23] World Health Organization, "Test procedures for insecticide resistance monitoring in malaria vectors, bio-efficacy and persistence of insecticides on treated surfaces," Repport of the WHO Informal Consultation, Document WHO/CDS/CPC/MAL/98.12. 5-22, Geneva, Switzerland, September 1998.

[24] D. J. Finney, Probit Analysis, Cambridge University Press, Cambidge, UK, 1971.

[25] R. Girod, E. Orlandi-Pradines, C. Rogier, and F. Pages, "Malaria transmission and insecticide resistance of Anopheles gambiae (Diptera: Culicidae) in the French military camp of Port-Bouet, Abidjan (Côte d'Ivoire): implications for vector control," Journal of Medical Entomology, vol. 43, no. 5, pp. 1082-1087, 2006.

[26] G. P. Georghiou and C. E. Taylor, "Pesticide resistance as an evolutionary phenomenon," in Proceedings of the 15th International Congress of Entomology, Entomological Society of America, Ed., pp. 759-785, Washington, DC, USA, 1976.

[27] V. Corbel, R. N'Guessan, C. Brengues et al., "Multiple insecticide resistance mechanisms in Anopheles gambiae and Culex quinquefasciatus from Benin, West Africa," Acta Tropica, vol. 101, no. 3, pp. 207-216, 2007.

[28] S. B. Vezenegho, B. D. Brooke, R. H. Hunt, M. Coetzee, and L. L. Koekemoer, "Malaria vector composition and insecticide susceptibility status in Guinea Conakry, West Africa," Medical and Veterinary Entomology, vol. 23, no. 4, pp. 326-334, 2009.

[29] S. Kumar, A. Thomas, A. Sahgal, A. Verma, T. Samuel, and M. K. K. Pillai, "Variations in the insecticide-resistance spectrum of Anopheles stephensi after selection with deltamethrin or a deltamethrin-piperonyl-butoxide combination," Annals of Tropical Medicine and Parasitology, vol. 98, no. 8, pp. 861-871, 2004.
[30] A. D. Stump, F. K. Atieli, J. M. Vulule, and N. J. Besansky, "Dynamics of the pyrethroid knockdown resistance allele in Western Kenyan populations of Anopheles gambiae in response to insecticide-treated bed net trials," American Journal of Tropical Medicine and Hygiene, vol. 70, no. 6, pp. 591-596, 2004. 


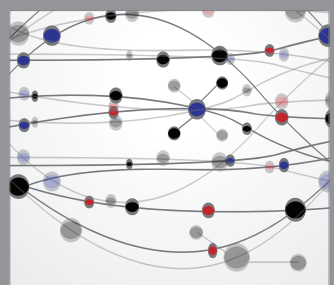

The Scientific World Journal
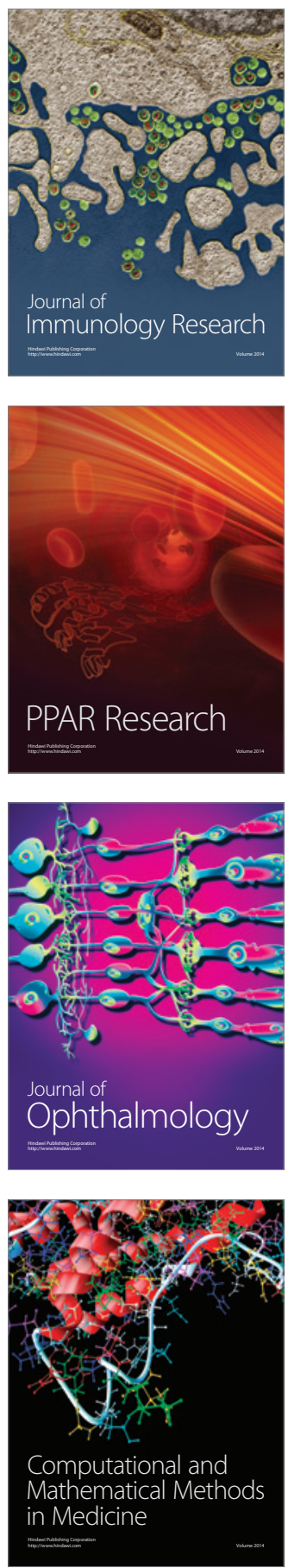

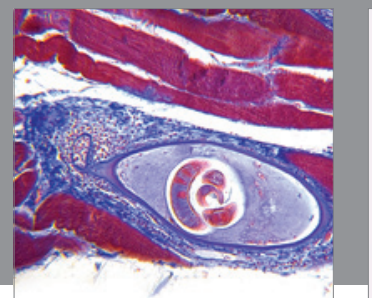

Gastroenterology

Research and Practice
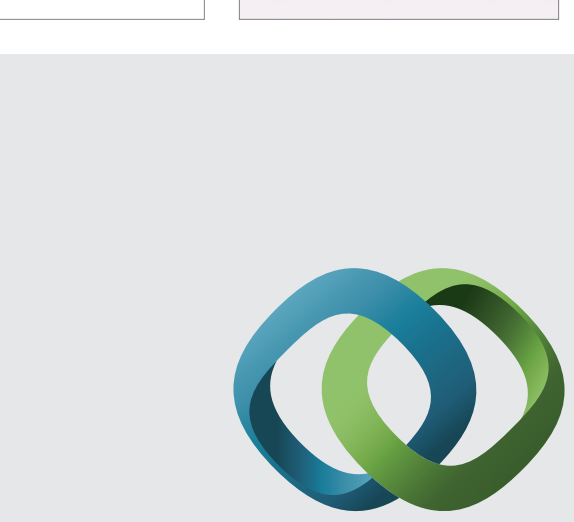

\section{Hindawi}

Submit your manuscripts at

http://www.hindawi.com
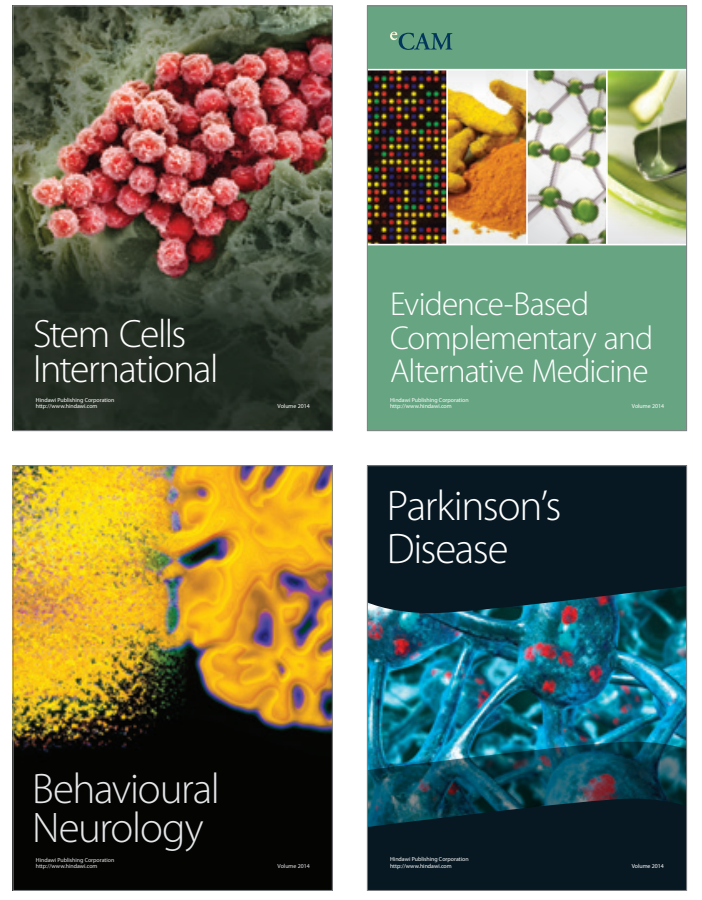
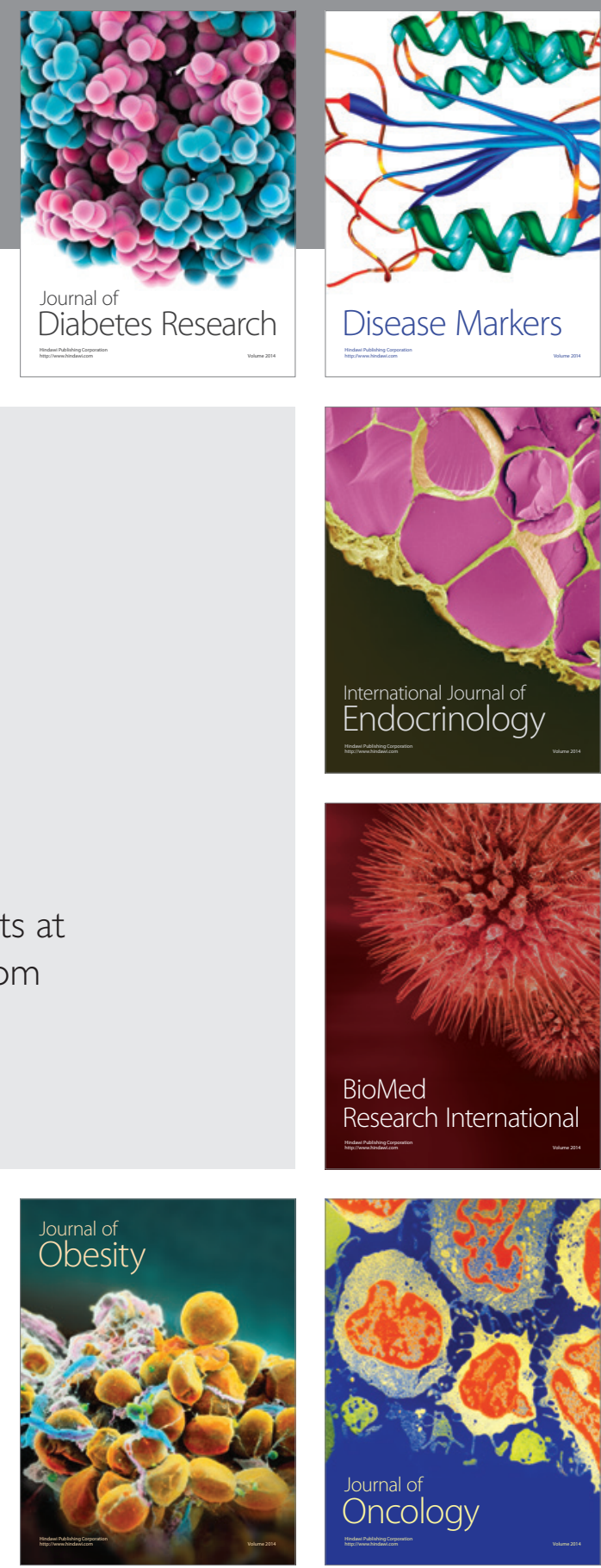

Disease Markers
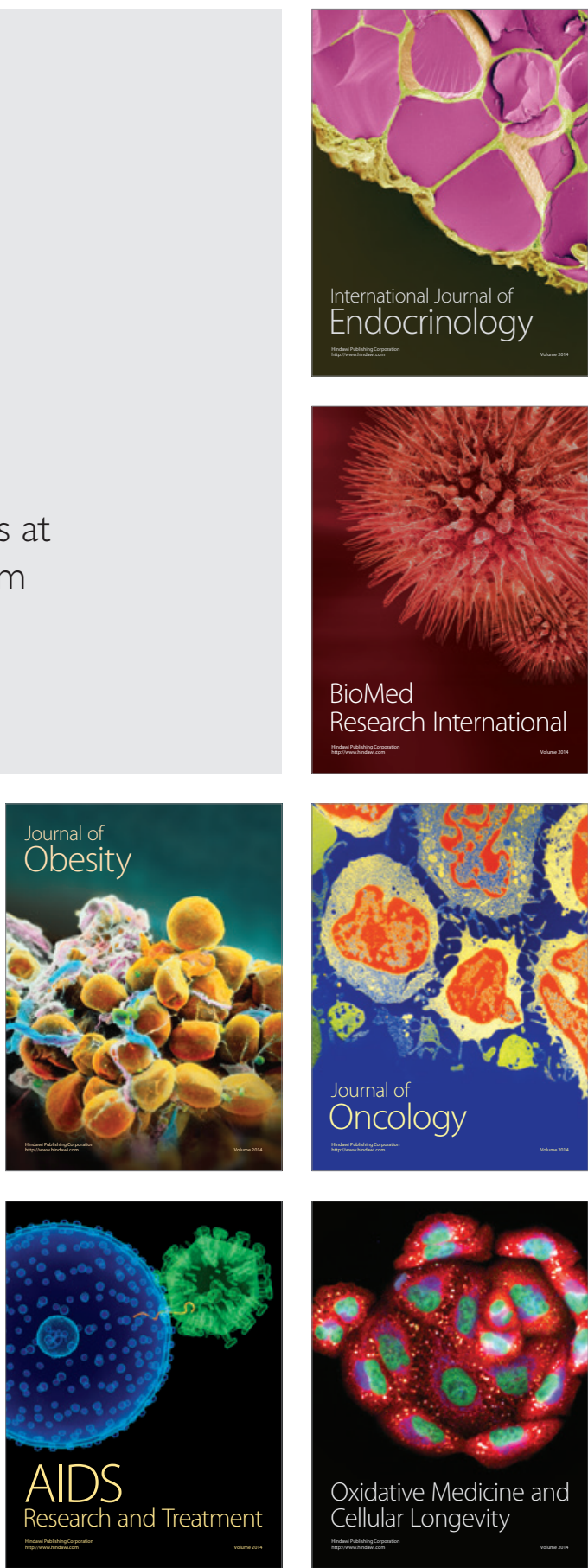